\title{
To Cath or Not to Cath: PCI for Chest Pain in Stable Coronary Artery Disease
}

\section{Mohamed Zakaria Khalil*}

Department of Medicine, Consultant Cardiologist, British Medical Center, Kuwait

${ }^{*}$ Corresponding author: Dr. Mohamed Zakaria Khalil, MD MRCP (UK), Professor, Department of Medicine, Consultant Cardiologist, British Medical Center, Kuwait Tel: +201100800262; E-mail: Prof.mzkhalil@gmail.com

Received date: March 30, 2018; Accepted date: April 04, 2018; Published date: April 09, 2018

Copyright: () 2018 Khalil MZ. This is an open-access article distributed under the terms of the Creative Commons Attribution License, which permits unrestricted use, distribution, and reproduction in any medium, provided the original author and source are credited.

\begin{abstract}
Most patients with symptomatic Coronary Artery Disease (CAD) are seeking quick relief for their angina by requesting Percutaneous Coronary Intervention $(\mathrm{PCI})$ that proved to be effective in Acute Coronary Syndrome (ACS), however, their request for $\mathrm{PCl}$ may be appropriately denied in stable CAD. Temptations for complete revascularization in CAD make certain valid target for patients as well as their treating physicians; nonetheless, cost effectiveness based on documented outcomes may not support interventional strategy.
\end{abstract}

Keywords: Coronary Artery; Chest pain; Clinical trials; Cardiac rest

\section{Introduction}

Chest pain as a result of CAD remains to be one of the most feared symptoms that bring patients to seek medical management. It has been made clear to public that anginal chest pain is an ominous symptom of cardiac origin indicative of heart attack, hence; urgent medical attention is advisable for evaluation of the possibility of Myocardial Infarction (MI) that may lead to sudden death.

Driven by the seriousness of their chest pain, patients typically present to the Emergency department of the nearest hospital for medical assessment. Ruling out a presumptive diagnosis of MI can be reached after thorough evaluation by standard protocols readily available in all hospitals. However, the persistence of chest pain despite absence of ACS may raise the potential necessity for referral to cathlab facility and plausible performance of PCI as an alternative swift management to Optimal Medical Therapy (OMT) [1-6]. The cost of PCI may not cause an obstacle when patients are paying from their own pocket, nonetheless, requesting approval from insurance companies are usually denied for coronary intervention in patients with stable CAD.

Evaluating patients with history of chest pain as a manifestation of CAD remains challenging, regardless of many insights and innovations over the past three decades. Obviously, CAD's chest pain is a product of reduced perfusion (ischemia) of a certain myocardial segment as a result of less blood supply facing a demand from the same segment. Therefore, the typical ischemic chest pain can easily be defined as an exertional chest pain, relieved by cardiac rest, and has the characteristics of angina.

Furthermore, relief of chest pain after nitroglycerin treatment does not predict active CAD, as it was found nonspecific for angina pectoris. Ischemic pain is generally described by patients vaguely as a discomfort rather than pain nonetheless; it is usually not felt as stabbing, or sharp pain. It is gradual in onset, however, its intensity may wax and wane, it radiates to other parts of the body characteristically to the upper extremities. The duration of the pain is typically used to classify either angina pectoris if it lasts less than twenty to thirty minutes, while the duration that lasts longer than 30 minutes is seen in patients with unstable angina as ACS.

Clinical evaluation of patients presenting with chest pain is further aided by a battery of investigations for reaching the diagnosis and initiating management [7]. Recurrence of chest pain requires optimization of medical therapy by aggressive risk factors management along with maximizing anti-anginal treatment as well as adopting recommended lifestyle modifications, nonetheless, compliance may represent an obstacle.

\section{Percutaneous coronary intervention (PCI) for CAD}

There is no evidence that PCI has significant survival benefit or prevent MI in patients with non-acute coronary artery syndrome compared with optimal medical therapy [7]. Chronic stable CAD is a diffuse disease process, making it more likely to respond to systemic treatment such as medical therapy compared to local therapy using PCI. On the other hand, PCI has definite survival benefit over medical therapy in patients with ACS or those who present with cardiogenic shock when performed by skilled operators in timely approach $[8,9]$

Early intervention relied mainly on balloon dilation (angioplasty), however, due to failure rate to maintain target vessel lesion patent, and to reduce target vessel revascularization (TVR); the use of stent was introduced to overcome such problem and became standard in most PCI procedures.

Despite successful revascularization performed by PCI to stable CAD, a number of patients, continue to suffer from chest pain. Recurrent angina is therefore defined as an experience of chest pain as a result of reduced blood supply to certain areas of the myocardium in a patient underwent prior revascularization procedure for CAD. It has been shown by several studies that nearly one third of patients reported recurrent angina following PCI [10-17].

Furthermore, microcirculation as a cause of angina is not tackled by PCI due to technical inability for interventions in patients with micro vascular dysfunction and structurally normal coronary arteries. 


\section{PCI placebo effect (I shall please)}

The word "placebo" originated from Latin, and it means "I shall please". The word 'Placebo' was first used medically in 1811, defined as "any medicine adapted more to please than to benefit the patient". In fact, placebos were commonly used in eighteen century medical practice, and were often endorsed as necessary deceptions. Doctors, back then in that era, were utilizing the powerful influence of patient's imagination to ease a disease that is not actually treated.

Clearly, the investigators of the ORBITA trial have provided robust evidence of the placebo effect gained from performing PCI to patients with stable CAD who have at least one significant lesion in single vessel disease [18]. In this trial, patients were randomized in two arms; either to receive actual revascularization PCI, or to receive sham (Fake) PCI. The results showed no significant statistical difference between the two arms, which can only be explained by a placebo effect of performing sham invasive procedure believed by patients to fix their underlying CAD.

It is important to emphasize that complaints of chest pain are basically subjective to patient description and perception. Objective pain assessment is lacking clinical investigation to measure the feeling of pain, hence; threshold of angina is variable patient's state of mind. Clearly, patient felt a relief of their stable angina following PCI as they felt reassured by the intervention, and this improvement is rather functional irrespective to structural morphology.

\section{Cost effective appropriate healthcare}

Third party payment of medical expenses incurred by providing therapy is commonly encountered between treating doctors and patients as provided by most modern healthcare systems. Insurance companies developed a system of approvals and claim revisions for making final decisions on requested charges as coverage of corresponding insured patients. Survival benefits as well as improving the quality of life may override any disagreement over cost effectiveness; nonetheless, cost is usually an issue for medical expenses that lack clear beneficial evidence.

Appropriately, patients with stable CAD are advised against PCI over OMT based on the results of several clinical trials that showed non-significant benefit of PCI over OMT either on the short term symptoms or the long-term prognosis. However, patients may suffer with extreme anxiety after knowing the extent of their CAD that can be fixed by PCI making one continue to wonder: should we consider a placebo effect of PCI as a necessary deception in 2018 ?

\section{References}

1. Coylewright M, Dick S, Zmolek B, Askelin J, Hawkins E, et al. (2016) PCI choice decision aid for stable coronary artery disease: A randomized trial. Circ Cardiovasc Qual Outcomes 9: 767-776.
2. Marwick TH, Cho I, Hartaigh Bó, Min JK (2015) Finding the gatekeeper to the cardiac catheterization laboratory: Coronary CT angiography or stress testing? J Am Coll Cardiol 65: 2747-2756.

3. Silva GV (2012) Appropriateness criteria for coronary revascularization in stable-angina patients. Coulter SA, ed. Tex Heart Inst J 39: 235-236.

4. Coylewright M, Shepel K, Leblanc A, Pencille L, Hess E, et al. (2012) Shared decision making in patients with stable coronary artery disease: PCI choice. PLoS One.7: e49827.

5. Epstein SE, Waksman R, Pichard AD, Kent KM, Panza JA (2013) Percutaneous coronary intervention versus medical therapy in stable coronary artery disease: The unresolved conundrum. JACC Cardiovasc Interv. 6: 993-8.

6. Coylewright M, O'Neill ES, Dick S, Grande SW (2017) PCI choice: Cardiovascular clinicians' perceptions of shared decision making in stable coronary artery disease. Patient Educ Couns. 100: 1136-1143.

7. Khalil MZ, Al Nozha MM (2012) Appropriate management of coronary artery disease. Adv Pharmacoepidem Drug Safety 1: 122.

8. Boden WE, O'Rourke RA, Teo KK, Hartigan PM, Maron DJ, et al. (2007) COURAGE trial research group. optimal medical therapy with or without PCI for stable coronary disease. N Engl J Med. 356: 1503-16.

9. Holper EM, Addo T (2010) Clinical implications of the BARI $2 \mathrm{D}$ and COURAGE trials: The evolving role of percutaneous coronary intervention. Coron Artery Dis 21: 397-401.

10. Abbate A, Biondi-Zoccai GG, Agostoni P, Lipinski MJ, Vetrovec GW (2007) Recurrent angina after coronary revascularization: A clinical challenge. Eur Heart J 28: 1057-65.

11. Lemos PA, Hoye A, Serruys PW (2004) Recurrent angina after revascularization: an emerging problem for the clinician. Coron Artery Dis 1: S11-5.

12. Thadani U (2004) Recurrent and refractory angina following revascularization procedures in patients with stable angina pectoris. Coron Artery Dis 1: S1-4.

13. Throndson K, Sawatzky JA (2009) Angina following percutaneous coronary intervention: In-stent restenosis. Can J Cardiovasc Nurs 19: 16-23.

14. Satran D, Traverse JH, Barsness GW, Lerman A, Simari RD, et al. Emerging therapies for refractory angina. Minn Med. 2008 Jan;91(1): 36-9.

15. Gori T, Fineschi M. (2007) Recurrent angina and the problem of inadequate/inappropriate revascularization. Eur Heart J 28: 2951.

16. Marzilli M (2004) Recurrent and resistant angina: Is the metabolic approach an appropriate answer? Coron Artery Dis. 1: S23-7.

17. Gersh BJ, Frye RL (2005) Methods of coronary revascularization--things may not be as they seem. N Engl J Med 352: 2235-7.

18. Al-Lamee R, Thompson D, Dehbi HM, Sen S, Tang K, et al. (2018) Percutaneous coronary intervention instable angina (ORBITA): A double-blind, randomised controlled trial. Lancet 391: 31-40. 